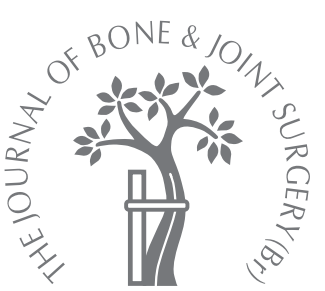

Y.-H. Kim, J.-S. Kim, D.-Y. Kim

From Ewha Womans University School of Medicine, Seoul, Korea

\title{
Clinical outcome and rate of complications after primary total knee replacement performed with quadriceps-sparing or standard arthrotomy
}

\begin{abstract}
We performed a prospective, randomised study to compare the results and rates of complications of primary total knee replacement performed using a quadriceps-sparing technique or a standard arthrotomy in 120 patients who had bilateral total knee replacements carried out under the same anaesthetic. The clinical results, pain scales, surgical and hospital data, post-operative complications and radiological results were compared.

No significant differences were found between the two groups with respect to the blood loss, knee score, function score, pain scale, range of movement or radiological findings. In contrast, the operating time $(p=0.0001)$ and the tourniquet time $(p<0.0001)$ were significantly longer in the quadriceps-sparing group, as was the rate of complications $(p=0.0468)$.

We therefore recommend the use of a standard arthrotomy with the shortest possible skin incision for total knee replacement.
\end{abstract}

In total knee replacement (TKR), the miniincision and quadriceps-sparing approaches were developed to minimise soft-tissue damage and enhance early rehabilitation. ${ }^{1-5}$ Tria $^{1}$ limited the medial parapatellar arthrotomy to the level of the superior pole of the patella and displaced the patella laterally without eversion. The early results indicated that there was reduced intra-operative blood loss, less pain, better early mobilisation and a shorter hospital stay. The accuracy of the alignment of the implant was found to be similar to that when using a standard incision. ${ }^{1-6}$ We have undertaken a prospective, randomised study in patients undergoing bilateral TKR. A quadriceps-sparing technique was used on one side and a standard arthrotomy on the other. We compared the differences in the surgical parameters, positioning of the component, safety and the rate of complications in each group.

\section{Patients and Methods}

Between August 2004 and January 2005, 132 consecutive patients underwent primary bilateral TKR undertaken under the same anaesthetic. Each operation was undertaken by the senior author (YHK). A total of 12 patients were lost to follow-up leaving 120 in the study. There were 27 men and 93 women with a mean age at the time of the operation of
65.4 years (43 to 88 ). Their mean weight was $61.8 \mathrm{~kg}$ (46 to 90) and mean height $152.6 \mathrm{~cm}$ (134 to $189 \mathrm{~cm})$. The mean body mass index was $28.1 \mathrm{~kg} / \mathrm{m}^{2}$ (19 to 36$)$. The pre-operative diagnosis was osteoarthritis in 113 patients $(94.2 \%)$, osteonecrosis in six $(5 \%)$ and rheumatoid arthritis in one $(0.8 \%)$. Epidural anaesthesia was used in 108 patients $(90 \%)$ and general anaesthesia in $12(10 \%)$. The mean follow-up was for 21.5 months (17 to 24$)$. The study was approved by our institutional review board and all patients gave informed consent.

The senior author performed approximately 25 TKRs using the quadriceps-sparing approach before starting the present study in order to eliminate bias due to the learning curve.

Each patient underwent TKR using the quadriceps-sparing technique in one knee and a standard arthrotomy in the other. Randomisation determined which knee was done first using a sequential pool on the basis of a table of randomised numbers.

In the quadriceps-sparing group, all the procedures were performed through an anterior midline skin incision measuring $\leq 10 \mathrm{~cm}$ in length. The extent of any incision into the quadriceps tendon was limited to the level of the superior pole of the patella. Vastus medialis was not split. Any extension of the incision into the quadriceps tendon proximally was 
Table I. The mean knee and functional scores in both groups

\begin{tabular}{|c|c|c|c|c|c|c|}
\hline & \multicolumn{2}{|c|}{ Quadriceps-sparing } & \multicolumn{2}{|c|}{ Standard arthrotomy } & \multicolumn{2}{|l|}{ p-value } \\
\hline & Knee score & $\begin{array}{l}\text { Functional } \\
\text { score }\end{array}$ & Knee score & $\begin{array}{l}\text { Functional } \\
\text { score }\end{array}$ & Knee score & Functional score \\
\hline Pre-operative & 27 (5 to 35$)$ & 49 (5 to 55$)$ & 28 (7 to 33$)$ & 53 (15 to 58$)$ & 0.607 & 1.000 \\
\hline \multicolumn{7}{|l|}{ Post-operative } \\
\hline 3 months & 85 (68 to 90$)$ & $76(55$ to 80$)$ & 83 (70 to 92$)$ & 73 (55 to 85$)$ & 0.3632 & 0.6728 \\
\hline 1 year & 90 (68 to 100$)$ & 81 (65 to 100$)$ & 91 (71 to 100$)$ & 82 (68 to 100$)$ & 0.2478 & 0.3158 \\
\hline 1.8 years ( 1.4 to 2 ) & 91 (71 to 100$)$ & 85 (85 to 100$)$ & 92 (76 to 100$)$ & 83 (81 to 100$)$ & 0.607 & 0.661 \\
\hline
\end{tabular}

considered to be an iatrogenic split of the tendon. The suprapatellar pouch was not opened, the patella was not everted and the suprapatellar fat pad was not resected.

In the standard arthrotomy group, all the procedures were performed through a similar skin incision of the same length, with a standard medial parapatellar arthrotomy of the joint. The extent of the incision of the quadriceps tendon was $4 \mathrm{~cm}$ to $8 \mathrm{~cm}$ proximal to the level of the superior pole of the patella depending on the size of the patient. The mobile upper aspect of the skin incision was used to expose the proximal portion of the quadriceps tendon. The suprapatellar pouch was opened and the patella everted but the suprapatellar fat pad was not resected.

The anterior and posterior cruciate ligaments were excised in all knees. The Nexgen Legacy Posterior Stabilising prosthesis (Zimmer, Warsaw, Indiana) was implanted using cement in all knees. In the quadriceps-sparing group, the four-in-one minimal invasive surgery quadricepssparing instruments (Zimmer) were used. These allowed femoral and tibial bone cuts to be made from the medial side of the knee. In the standard arthrotomy group, the same instruments were used, but the femoral and tibial bone cuts were made from the front rather than from the side of the knee. In all cases, ligamentous balancing was performed. After pulsed lavage, drying and pressurisation, the cemented implants were inserted.

After surgery, an epidural block was maintained for two days. The patients received a basal level of analgesics and were allowed to increase this amount by using a selfadministered patient-controlled analgesia pump.

The knees were splinted in $15^{\circ}$ of flexion for the first 24 hours after the operation and then mobilisation was commenced using a continuous-passive motion machine. An active and passive range-of-movement programme and walking, taking weight as pain allowed, was begun on the second day. The patients used a walking stick for 12 weeks.

Clinical and radiological follow-up was undertaken at three months, one year and annually thereafter. This follow-up was performed by one of the authors (DYK) who was blinded to the type of arthrotomy used. Each knee was rated pre-operatively and post-operatively according to the Knee Society scoring (KSS) system. ${ }^{7}$ Patients evaluated their knee pain subjectively using a ten-point visual analogue scale, with 0 points indicating no pain and 10 severe pain.
The data collected for analysis were the operating time, tourniquet time, intra-operative blood loss, blood replacement during hospitalisation, the amount of drainage, the mean duration of drainage and the length of hospital stay. Homologous transfusion was performed when the haemoglobin level fell below $10.0 \mathrm{~g} / \mathrm{dl}$ and the patient had symptoms including dizziness, headache and nausea. The drains were removed when the amount of drainage was less than $50 \mathrm{ml}$ over 48 hours. Complications were recorded.

Standing and supine anteroposterior and lateral radiographs, and patellar skyline views were the basis for all preoperative and post-operative assessment. Limb alignment, the position of the components and the presence and location of radiolucent lines at the cement-bone interface according to the recommendation of the Knee Society were assessed. ${ }^{7}$ The level of the joint line was determined preand post-operatively. The radiological measurements were taken using a picture archiving and communication system (PACS; Pixview Starr, Infinitt Co., Seoul, Korea). Three measurements were performed on each radiograph by an observer who was not an author.

Statistical analysis. This was done using a two-tailed $t$-test for continuous variables and a chi-squared contingency table for dichotomous variables. A p-value of $\leq 0.05$ was considered to be significant.

It was calculated that at least 55 patients would be required in order to determine whether there was a significant difference (power, 0.8, p < 0.05) in the Knee Society score, surgical and hospital data, post-operative complications and radiological results between the two groups.

\section{Results}

Clinical findings. The mean pre-operative KSS was 27 points $(5$ to 35$)$ in the quadriceps-sparing group and 28 (7 to 33 ) in the SA group ( $\mathrm{p}=0.607)$. The mean pre-operative functional score was 49 points ( 5 to 55 ) in the quadricepssparing group and 53 (15 to 58) in the standard arthrotomy group ( $\mathrm{p}=1.000)$. At the final follow-up the mean KSS was 91 points (71 to 100$)$ in the quadriceps-sparing group and 92 points (76 to 100$)$ in the standard arthrotomy group $(\mathrm{p}=0.667)$. In the quadriceps-sparing group the mean functional score at the latest follow-up was 85 points and 83 points in the standard arthrotomy group $(\mathrm{p}=0.661$ ) (Table I). The post-operative pain scales in the two groups 
Table II. Operative and hospital data (range) for both groups

\begin{tabular}{|c|c|c|c|}
\hline & Quadriceps-sparing & Standard arthrotomy & p-value \\
\hline Operating time (min) & $95.6(60$ to 120$)$ & 89.5 (55 to 122 ) & 0.0001 \\
\hline Tourniquet time (min) & 62.8 (45 to 98 ) & 54.5 (39 to 80$)$ & $<0.0001$ \\
\hline Intra-operative blood loss (ml) & 359.0 (100 to 950$)$ & $336.4(120$ to 1080$)$ & 0.400 \\
\hline Haemovac drainage (ml) & 1112.5 (240 to 2920 ) & 1076.2 (300 to 1630 ) & 0.012 \\
\hline Duration of haemovac drains (days) & 5.1 (2 to 6$)$ & 4.9 (2 to 7$)$ & 0.246 \\
\hline \multicolumn{4}{|l|}{ Length of skin incision $(\mathrm{cm})$} \\
\hline Extension & 10.5 (7.5 to 22.0$)$ & 10.8 (8.5 to 12.5$)$ & 0.288 \\
\hline Flexion & $12.4(10$ to 14$)$ & $12.5(10$ to 15$)$ & 0.798 \\
\hline
\end{tabular}

Table III. Mean radiological findings (range, SD) for both groups

\begin{tabular}{|c|c|c|c|c|c|c|c|}
\hline \multirow[b]{2}{*}{ Knee alignment (valgus) $\left({ }^{\circ}\right)$} & \multicolumn{3}{|c|}{ Quadriceps-sparing } & \multicolumn{3}{|c|}{ Standard arthrotomy } & \multirow{2}{*}{$\begin{array}{l}\text { p-value } \\
0.1334\end{array}$} \\
\hline & 5.5 & $(-3$ to 9$)$ & 2.6 & 5.2 & $(-2$ to 11$)$ & 2.2 & \\
\hline \multicolumn{8}{|l|}{ Femoral component position $\left({ }^{\circ}\right)$} \\
\hline Coronal plane & 98.3 & (95 to 101$)$ & 1.9 & 96.8 & (93 to 98$)$ & 2.2 & 0.3524 \\
\hline Sagittal plane & 2.7 & $(-3$ to 9$)$ & 3.2 & 2.5 & $(-4$ to 10$)$ & 2.4 & 0.7349 \\
\hline \multicolumn{8}{|l|}{ Tibial component position $\left({ }^{\circ}\right)$} \\
\hline Coronal plane & 89 & (95 to 91$)$ & 3 & 87 & (82 to 95$)$ & 2.3 & 0.8923 \\
\hline Sagittal plane & 87 & (79 to 89 ) & 2 & 85 & (80 to 93 ) & 2.2 & 0.9238 \\
\hline Patellar component angle $\left({ }^{\circ}\right)$ & 5 & $(-9$ to 18$)$ & 3.7 & 4.1 & $(-10$ to 21$)$ & 4.8 & 0.2673 \\
\hline Tibial surface capping (\%) & 98 & (90 to 105$)$ & 2.1 & 97 & (91 to 105$)$ & -2.5 & 0.7435 \\
\hline \multicolumn{8}{|l|}{ Joint line $(\mathrm{cm})$} \\
\hline Pre-operative & 16.1 & (6 to 21$)$ & 4.3 & 16.3 & (5 to 25$)$ & 4.1 & 0.8612 \\
\hline Post-operative & 15.8 & (8 to 24$)$ & 4.1 & 15.7 & (9 to 23 ) & 3.7 & 0.5134 \\
\hline \multicolumn{8}{|l|}{ Posterior condylar offset $(\mathrm{cm})$} \\
\hline Pre-operative & 24.1 & (18 to 31 ) & 2.2 & 24.9 & (17 to 29$)$ & 3.1 & 0.6233 \\
\hline Post-operative & 24.9 & (21 to 32 ) & 2.5 & 26.3 & (17 to 31$)$ & 2.8 & 0.8112 \\
\hline
\end{tabular}

at three months, one year, and a mean of 1.8 years (1.4 to 2 ) post-operatively were not significantly different $(\mathrm{p}>0.05)$.

At the three-month follow-up the mean range of movement was $128^{\circ}\left(110^{\circ}\right.$ to $\left.135^{\circ}\right)$ in the quadriceps-sparing group and $129^{\circ}\left(108^{\circ}\right.$ to $\left.140^{\circ}\right)$ in the standard arthrotomy group. This difference was not significant $(\mathrm{p}=0.549)$. At the final follow-up, the mean range of movement was $130^{\circ}$ $\left(110^{\circ}\right.$ to $\left.150^{\circ}\right)$ in the quadriceps-sparing group and $130.6^{\circ}$ $\left(110^{\circ}\right.$ to $\left.145^{\circ}\right)$ in the standard arthrotomy group, which again was not significant $(\mathrm{p}=0.531)$. The range of movement was measured clinically with a protractor.

The mean length of the final skin incision with the knee in $90^{\circ}$ of flexion was $12.4 \mathrm{~cm}$ (10 to 14$)$ in the quadricepssparing group and $12.5 \mathrm{~cm}$ (10 to 15$)$ in the standard arthrotomy group. This difference was not significant $(\mathrm{p}=$ 0.798, Table II). In the quadriceps-sparing group 31 $(25.8 \%)$ knees had iatrogenic laceration of the quadriceps tendon approximately $2.5 \mathrm{~cm}$ in length because of excessive retraction to achieve adequate exposure.
The mean operating time and the tourniquet time were significantly longer in the quadriceps-sparing group $(\mathrm{p}=$ $0.0001, \mathrm{p}<0.0001$ respectively; Table II). By contrast, the mean intra-operative blood loss, the mean amount of drainage, and the mean duration of the use of suction drains were not significantly different $(\mathrm{p}>0.05)$ (Table II). The mean pre-operative haemaglobin and haematocrit values were $13.8 \mathrm{~g} / \mathrm{dl}$ (11 to 16 ) and $38.9 \%$ (29.8\% to $45.1 \%$ ), respectively. The mean post-operative haemoglobin and haematocrit values at the time of discharge were $10.9 \mathrm{~g} / \mathrm{dl}$ (9.1 to 13.9 ) and $32.8 \%(24.9 \%$ to $41.2 \%)$, respectively. The mean length of hospital stay for both groups was 12.9 days (7 to 15$)$. All the patients were discharged directly to their homes.

A total of 16 quadriceps-sparing procedures (13\%) and seven standard arthrotomy procedures $(6 \%)$ were associated with an operative or post-operative complication $(p=0.468)$. In the quadriceps-sparing group, two knees $(1.7 \%)$ had laceration of the popliteus tendon, two $(1.7 \%)$ had palsy of the deep peroneal nerve, which 
resolved spontaneously within five and six months and one $(1 \%)$ had a partial avulsion of the patellar tendon. Five knees $(4 \%)$ had notching of the anterior femoral condylar and one knee $(1 \%)$ had a supracondylar fracture. This required open reduction and internal fixation by a plate and screws. One knee $(1 \%)$ had a medial epicondylar avulsion fracture which was fixed by a screw. One knee $(1 \%)$ developed a superficial infection which resolved after intravenous antibiotics for six weeks. Two knees (1.7\%) developed a deep infection which resolved after open debridement and intravenous antibiotics for six weeks. One knee $(1 \%)$ developed a deep vein thrombosis (DVT) in the calf vein. The patient was not treated for DVT and there were no further complications.

A total of 31 knees $(25.8 \%)$ had laceration of the quadriceps tendon inadvertently $2.5 \mathrm{~cm}$ in length along the substance of the quadriceps tendon, proximally from the superior pole of the patella. The laceration of the quadriceps tendon did not affect the post-operative management.

In the standard arthrotomy group, two knees $(1.7 \%)$ had anterior femoral notching. One $(1 \%)$ sustained an undisplaced fracture of the posteromedial tibial plateau which was fixed by a screw, and two others $(1.7 \%)$ had a medial epicondylar avulsion fracture, also fixed by screws. One knee $(1 \%)$ developed a superficial infection which resolved after intravenous antibiotics for three weeks, and one $(1 \%)$ developed a deep infection which resolved after open debridement and intravenous antibiotics for six weeks.

Radiological findings. These are summarised in Table III. There were no significant differences between the groups with regard to the position of the femoral and tibial components in the coronal and sagittal planes, the alignment of the knees, the patellar angle, the tibial surface area covered by the implants, the level of the joint line and the posterior femoral condylar offset $(\mathrm{p}>0.05)$. No knee in either group had evidence of radiolucent lines around any component. There was no evidence of aseptic loosening of the femoral, tibial or patellar components, or patellar subluxation or dislocation in either group.

\section{Discussion}

Early reports of the use of a mini-incision and quadriceps sparing approach for TKR indicated that there was reduced intra-operative blood loss, less pain, better early movement, and a shorter hospital stay, with an accuracy of the alignment of the implant similar to that of TKR with a standard incision. ${ }^{1-6}$ In our study, the early complication rate was significantly higher in the quadriceps-sparing group. Major complications included one superficial and two deep infections, two cases of palsy of the peroneal nerve, and one supracondylar fracture. We believe that the higher rate of complications in the quadriceps-sparing group was related to limited visualisation. Working through a small incision may cause more pressure on the skin and soft tissues from stronger retraction. There may be more abrasion of the skin edges from reamers and rasps. This may result in more wound complications and infections. The operative and tourniquet times were significantly longer in the quadriceps-sparing group.

Considerable effort was made to preserve the quadriceps tendon in the quadriceps-sparing group, but no significant differences were found between the groups with respect to the knee score, pain scale, range of movement, hospital data or radiological parameters. We believe that because there were no short-term differences in these parameters the long-term results may also be similar.

It was very difficult to place a stemmed tibial component using the quadriceps-sparing approach and the quadriceps tendon was lacerated inadvertently in 31 knees $(25.8 \%)$. Because the benefit of the quadriceps-sparing technique was negligible and the rate of complications higher, we suggest that a formal incision of the quadriceps tendon $2 \mathrm{~cm}$ to $4 \mathrm{~cm}$ proximal to the superior pole of the patellar should be an important part of the exposure.

A weakness of our study may be attributed to potential bias in the assessment of the clinical and radiological results, variables in the type of anaesthetic used and in the type of pathology encountered.

We routinely perform bilateral TKR under a single anaesthesia session unless patients have serious medical problems. To date we have experienced no major systemic complications such as fatal pulmonary emboli in a total series of more than 2000 patients treated in this way.

All patients in both groups favoured a short skin incision. Despite there being no differences in most of the clinical and radiological outcome parameters between the two groups, the operating time was longer and the early complication rate higher in the quadriceps-sparing group. We therefore recommend a standard arthrotomy with the shortest possible skin incision when performing TKR.

The authors would like to thank Sang-Mi Lee for collecting and analysing data for this manuscript.

No benefits in any form have been received or will be received from a commercial party related directly or indirectly to the subject of this article.

\section{References}

1. Tria AJ Jr. Minimally invasive total knee arthroplasty: the importance of instrumentation. Orthop Clin North Am 2004;35:227-34

2. Tria AJ Jr, Coon TM. Minimal incision total knee arthroplasty: early experience. Clin Orthop 2003;416:185-90

3. Tria AJ Jr. Advancements in minimally invasive total knee arthroplasty. Orthopedics 2003;26(8 Suppl):859-63.

4. Bonutti PM, Mont MA, Kester MA. Minimally invasive total knee arthroplasty: a 10-feature evolutionary approach. Orthop Clin North Am 2004;35:217-26.

5. Laskin RS, Beksac B, Phongjunakorn A, et al. Minimally invasive total knee replacement through a mini-midvastus incision: an outcome study. Clin Orthop 2004; $428: 74-81$

6. Scuderi GR, Tria AJ Jr. Minimal incision total knee arthroplasty. In: Scuderi GR, Tria AJ Jr, eds. MIS of the hip and the knee: a clinical perspective. London: Springer, 2004:175-86.

7. Ewald FC. The Knee Society total knee arthroplasty roentgenographic evaluation and scoring system. Clin Orthop 1989;248:9-12. 